\title{
Is Usnic Acid a Promising Radical Scavenger?
}

Nguyen Thi Hoa ${ }^{1}$, May Van Bay ${ }^{2}$, Adam Mechler ${ }^{3}$ and Quan V. Vo ${ }^{4,5^{*}}$

${ }^{1}$ Academic Affairs, The University of Danang - University of Technology and Education, 48 Cao Thang, Danang 550000, Vietnam.

${ }^{2}$ Department of Chemistry, The University of Danang - University of Science and Education, Danang 550000, Vietnam.

${ }^{3}$ Department of Chemistry and Physics, La Trobe University, Victoria 3086, Australia ${ }^{4}$ Institute of Research and Development, Duy Tan University, Danang 550000, Vietnam.

${ }^{5}$ Faculty of Chemical Technology - Environment, The University of Danang - University of Technology and Education, 48 Cao Thang, Danang 550000, Vietnam.

*Corresponding author: vovanquan2@duytan.edu.vn; vvquan@ute.udn.vn

\section{List of supporting information:}

Table of Contents

Table S1: Cartesian coordinates of TS in water and pentyl ethanoate. 
Table S1: Cartesian coordinates of TS in water and pentyl ethanoate.

\begin{tabular}{|c|c|c|c|c|c|c|c|}
\hline Water & & & & $\mathrm{Pe}$ & Ianoate & & \\
\hline Name: & -C10-H-OH-FH & & & & & & \\
\hline & & & & oor & & & \\
\hline $\mathrm{O}$ & 0.23012100 & 1.98662200 & 0.23973200 & 0 & 0.21146300 & 1.94143500 & 0.22450700 \\
\hline 0 & -2.04060000 & -1.94241000 & -0.23305400 & 0 & -2.01486200 & -2.00173900 & -0.28640100 \\
\hline $\mathrm{O}$ & 0.39167000 & -2.66363700 & -0.06714100 & $\mathrm{O}$ & 0.54188900 & -2.71164100 & -0.06220500 \\
\hline 0 & -4.49230500 & 2.12661800 & 0.07589800 & $\mathrm{O}$ & -4.49954300 & 2.00824800 & -0.01048300 \\
\hline 0 & 4.41901700 & -0.28296200 & -0.65009200 & $\mathrm{O}$ & 4.46974500 & -0.19247700 & -0.61129500 \\
\hline 0 & -5.64977700 & -0.14354000 & -0.93979700 & $\mathrm{O}$ & -5.70779400 & -0.01568300 & -0.40892200 \\
\hline 0 & 4.43563500 & 2.19973900 & -0.48567600 & 0 & 4.40634400 & 2.27895700 & -0.49580900 \\
\hline $\mathrm{C}$ & -1.01299500 & 0.01898200 & 0.58162400 & $\mathrm{C}$ & -0.97378300 & -0.05149300 & 0.56546200 \\
\hline $\mathrm{C}$ & 0.37084300 & -0.28842400 & 0.06251000 & $\mathrm{C}$ & 0.42135900 & -0.32948500 & 0.06065200 \\
\hline $\mathrm{C}$ & -1.05317600 & 1.48597400 & 0.32319600 & $\mathrm{C}$ & -1.04356600 & 1.42334600 & 0.32077800 \\
\hline $\mathrm{C}$ & -2.20789600 & -0.71188800 & 0.00857900 & $\mathrm{C}$ & -2.15203400 & -0.80216900 & -0.01885100 \\
\hline $\mathrm{C}$ & -3.42810100 & -0.00569000 & -0.11873900 & $\mathrm{C}$ & -3.39552200 & -0.07270000 & -0.09711600 \\
\hline $\mathrm{C}$ & 1.06224900 & 0.90463700 & 0.00706200 & C & 1.07857300 & 0.88196500 & 0.00220700 \\
\hline $\mathrm{C}$ & 1.04761700 & -1.49641600 & -0.12763700 & $\mathrm{C}$ & 1.14053000 & -1.51771200 & -0.11932600 \\
\hline $\mathrm{C}$ & -2.16802300 & 2.18616400 & 0.14697200 & $\mathrm{C}$ & -2.17141500 & 2.12821600 & 0.18231400 \\
\hline $\mathrm{C}$ & -3.44587300 & 1.44801900 & 0.05308100 & $\mathrm{C}$ & -3.41032400 & 1.37711200 & 0.05647200 \\
\hline $\mathrm{C}$ & 2.43961200 & 1.00321200 & -0.23048100 & $\mathrm{C}$ & 2.44964700 & 1.02620500 & -0.22827400 \\
\hline $\mathrm{C}$ & 2.42791200 & -1.48237400 & -0.39938900 & $\mathrm{C}$ & 2.51998200 & -1.45904200 & -0.37571100 \\
\hline $\mathrm{C}$ & 3.09153400 & -0.24988300 & -0.42671900 & $\mathrm{C}$ & 3.14666600 & -0.20582400 & -0.40491800 \\
\hline $\mathrm{C}$ & -4.66453500 & -0.71820000 & -0.44833100 & $\mathrm{C}$ & -4.62314200 & -0.71738000 & -0.35732700 \\
\hline $\mathrm{C}$ & 3.20463400 & 2.24347700 & -0.27370200 & $\mathrm{C}$ & 3.17985500 & 2.29208000 & -0.29318400 \\
\hline $\mathrm{C}$ & 3.19949400 & -2.72925500 & -0.62804200 & $\mathrm{C}$ & 3.33092600 & -2.68353700 & -0.59083500 \\
\hline $\mathrm{C}$ & -4.79380000 & -2.19785500 & -0.17599800 & C & -4.79497600 & -2.17787600 & -0.57756700 \\
\hline $\mathrm{C}$ & 2.55139700 & 3.57206700 & -0.06729100 & $\mathrm{C}$ & 2.48324200 & 3.60668800 & -0.12125100 \\
\hline $\mathrm{H}$ & -2.18721100 & 3.25764000 & -0.00024800 & $\mathrm{H}$ & -2.19792200 & 3.20147900 & 0.05482600 \\
\hline $\mathrm{H}$ & 3.82291500 & -2.68555500 & -1.52060600 & $\mathrm{H}$ & 4.08212600 & -2.80554200 & 0.29417700 \\
\hline $\mathrm{H}$ & 3.94953600 & -2.87870300 & 0.23701500 & $\mathrm{H}$ & 2.73082900 & -3.58976600 & -0.59601100 \\
\hline $\mathrm{H}$ & 2.57315300 & -3.61773900 & -0.63390700 & $\mathrm{H}$ & 3.96491200 & -2.62207400 & -1.47477400 \\
\hline $\mathrm{H}$ & -0.60983500 & -2.45848900 & -0.07775500 & $\mathrm{H}$ & -0.43927900 & -2.58275600 & -0.09392000 \\
\hline $\mathrm{H}$ & -5.85535400 & -2.41969300 & -0.07028500 & $\mathrm{H}$ & -5.85872100 & -2.39689900 & -0.64084200 \\
\hline $\mathrm{H}$ & -4.40071400 & -2.75974200 & -1.02571400 & $\mathrm{H}$ & -4.29410600 & -2.47270800 & -1.50137000 \\
\hline $\mathrm{H}$ & -4.24548600 & -2.50802600 & 0.71149300 & $\mathrm{H}$ & -4.32841000 & -2.74466900 & 0.22807300 \\
\hline $\mathrm{H}$ & 4.72218800 & 0.67674900 & -0.63659800 & $\mathrm{H}$ & 4.73311300 & 0.78329600 & -0.61548100 \\
\hline $\mathrm{H}$ & 2.06793400 & 3.60654200 & 0.91007500 & $\mathrm{H}$ & 2.01473000 & 3.66170600 & 0.86296800 \\
\hline $\mathrm{H}$ & 1.78183300 & 3.73474200 & -0.82342200 & $\mathrm{H}$ & 1.69641400 & 3.72173600 & -0.86836400 \\
\hline $\mathrm{H}$ & 3.31297300 & 4.34568100 & -0.13582400 & $\mathrm{H}$ & 3.21994200 & 4.39986000 & -0.22782000 \\
\hline $\mathrm{C}$ & -1.05083000 & -0.23362600 & 2.11928400 & $\mathrm{H}$ & -5.40094800 & 0.99449600 & -0.24441600 \\
\hline $\mathrm{H}$ & -2.01201000 & 0.08148400 & 2.52758900 & $\mathrm{C}$ & -1.03514700 & -0.31186400 & 2.10438400 \\
\hline $\mathrm{H}$ & -0.90090400 & -1.29780100 & 2.30646200 & $\mathrm{H}$ & -2.00903700 & -0.02342400 & 2.50307800 \\
\hline $\mathrm{H}$ & -0.24868000 & 0.33124500 & 2.59616800 & $\mathrm{H}$ & -0.86144800 & -1.37224900 & 2.29282700 \\
\hline 0 & 5.14671800 & -2.55770000 & 1.12171900 & $\mathrm{H}$ & -0.25521200 & 0.26830600 & 2.59908700 \\
\hline $\mathrm{H}$ & 5.28547000 & -1.68371900 & 0.70699100 & $\mathrm{O}$ & 5.20883800 & -2.46400600 & 1.15761500 \\
\hline & & & & $\mathrm{H}$ & 5.36914700 & -1.60472200 & 0.72446000 \\
\hline Name: & -C12-H-OH-FH & & & & & & \\
\hline & & & & oor & & & \\
\hline $\mathrm{O}$ & -0.97139900 & -1.86488200 & 0.30302900 & 0 & -0.97872200 & -1.83599800 & 0.27482700 \\
\hline $\mathrm{O}$ & 1.91469400 & 1.59600600 & -0.41341800 & $\mathrm{O}$ & 1.91628400 & 1.65586800 & -0.31991900 \\
\hline 0 & -0.35487400 & 2.74473600 & -0.09173300 & $\mathrm{O}$ & -0.49098900 & 2.80550700 & -0.03690400 \\
\hline 0 & 3.63188700 & -2.83670700 & -0.10690500 & $\mathrm{O}$ & 3.60381600 & -2.76307900 & -0.20842400 \\
\hline 0 & -4.75731500 & 1.08758900 & -0.40961300 & $\mathrm{O}$ & -4.82431500 & 1.03564300 & -0.37974600 \\
\hline $\mathrm{O}$ & 5.13356900 & -0.84644400 & -1.21420300 & $\mathrm{O}$ & 5.14895900 & -1.01048600 & -0.71712800 \\
\hline 0 & -5.18060700 & -1.37234700 & -0.22117400 & $\mathrm{O}$ & -5.19781000 & -1.43204300 & -0.23912300 \\
\hline $\mathrm{C}$ & 0.61154400 & -0.13736500 & 0.51030600 & $\mathrm{C}$ & 0.55695400 & -0.08915800 & 0.51488300 \\
\hline
\end{tabular}




\begin{tabular}{|c|c|c|c|c|c|c|c|}
\hline C & -0.73209100 & 0.39889200 & 0.07704000 & c & -0.79155700 & 0.43669900 & 0.08702900 \\
\hline C & 0.37806300 & -1.59377300 & 0.29875000 & C & 0.34174400 & -1.55044800 & 0.30324700 \\
\hline C & 1.87512500 & 0.35636700 & -0.15961900 & C & 1.83334300 & 0.43287900 & -0.11034300 \\
\hline C & 2.94473500 & -0.55508100 & -0.33528300 & C & 2.90523800 & -0.51043000 & -0.27107200 \\
\hline C & -1.61921700 & -0.65565600 & 0.09095500 & C & -1.65814700 & -0.63371800 & 0.08196300 \\
\hline C & -1.20951100 & 1.70504200 & -0.08894400 & C & -1.30170300 & 1.73721200 & -0.05473800 \\
\hline C & 1.34211900 & -2.48332800 & 0.08549900 & C & 1.32730300 & -2.44478300 & 0.13355000 \\
\hline C & 2.71994800 & -1.98700900 & -0.10258600 & C & 2.64187000 & -1.90368500 & -0.08725500 \\
\hline C & -3.00412400 & -0.51666100 & -0.06696700 & C & -3.04289700 & -0.53163000 & -0.07269800 \\
\hline C & -2.58227400 & 1.93187900 & -0.25724200 & C & -2.67699600 & 1.92952600 & -0.21675600 \\
\hline C & -3.44347300 & 0.83119900 & -0.23972100 & C & -3.51853500 & 0.80815900 & -0.21889700 \\
\hline C & 4.26079300 & -0.08822800 & -0.76158600 & C & 4.25309600 & -0.13174100 & -0.64449600 \\
\hline C & -3.96427600 & -1.61414100 & -0.06854500 & C & -3.98401500 & -1.65303500 & -0.10053700 \\
\hline C & -3.14665700 & 3.31075800 & -0.46162000 & C & -3.27328400 & 3.29789200 & -0.39425800 \\
\hline C & 4.64708100 & 1.36481700 & -0.65633900 & C & 4.64619900 & 1.26992500 & -0.94112200 \\
\hline C & -3.52860600 & -3.03471400 & 0.10806600 & C & -3.51425800 & -3.07099600 & 0.03123300 \\
\hline $\mathrm{H}$ & 1.16451400 & -3.54475700 & -0.02373300 & $\mathrm{H}$ & 1.16946500 & -3.50978900 & 0.03835700 \\
\hline $\mathrm{H}$ & -3.68526100 & 3.37259500 & -1.40975500 & $\mathrm{H}$ & -3.97094500 & 3.52284600 & 0.41557700 \\
\hline $\mathrm{H}$ & -3.85162400 & 3.56355200 & 0.33348800 & $\mathrm{H}$ & -2.49275600 & 4.05513600 & -0.40411300 \\
\hline $\mathrm{H}$ & -2.35221600 & 4.05338700 & -0.46677900 & $\mathrm{H}$ & -3.83268200 & 3.35549500 & -1.33026400 \\
\hline $\mathrm{H}$ & 0.58446500 & 2.37230400 & -0.16613600 & $\mathrm{H}$ & 0.44441300 & 2.50248600 & -0.10855700 \\
\hline H & 5.72807600 & 1.46225000 & -0.73960000 & $\mathrm{H}$ & 5.68661800 & 1.31487600 & -1.25406900 \\
\hline $\mathrm{H}$ & 4.12515100 & 1.99834300 & -1.36959000 & $\mathrm{H}$ & 3.96622300 & 1.78806000 & -1.61110100 \\
\hline $\mathrm{H}$ & 4.36248300 & 1.77728500 & 0.39928000 & $\mathrm{H}$ & 4.59432600 & 1.87223400 & 0.07920200 \\
\hline $\mathrm{H}$ & -5.21845400 & 0.20024700 & -0.37568000 & $\mathrm{H}$ & -5.26699200 & 0.13876100 & -0.35998000 \\
\hline $\mathrm{H}$ & -3.01714800 & -3.15628900 & 1.06393500 & $\mathrm{H}$ & -3.00386100 & -3.21849900 & 0.98417500 \\
\hline $\mathrm{H}$ & -2.82899300 & -3.31533800 & -0.68063100 & $\mathrm{H}$ & -2.80845200 & -3.31505400 & -0.76431300 \\
\hline $\mathrm{H}$ & -4.40938900 & -3.67185800 & 0.07156000 & $\mathrm{H}$ & -4.38335400 & -3.72247700 & -0.02886900 \\
\hline C & 0.81214200 & 0.14133100 & 2.03148400 & $\mathrm{H}$ & 4.46322700 & -2.18219900 & -0.43631700 \\
\hline $\mathrm{H}$ & 1.73200200 & -0.32963100 & 2.38109400 & C & 0.74622300 & 0.15435800 & 2.05176800 \\
\hline $\mathrm{H}$ & 0.86745700 & 1.21969200 & 2.18940000 & $\mathrm{H}$ & 1.67173800 & -0.30380700 & 2.40284900 \\
\hline $\mathrm{H}$ & -0.03803800 & -0.26095800 & 2.58375600 & $\mathrm{H}$ & 0.77732500 & 1.22903400 & 2.23574800 \\
\hline 0 & 3.80483300 & 2.44106900 & 1.53304900 & $\mathrm{H}$ & -0.10095200 & -0.27408500 & 2.58814200 \\
\hline $\mathrm{H}$ & 2.90275300 & 2.42103400 & 1.15524400 & 0 & 4.18431900 & 2.64061000 & 1.14391100 \\
\hline & & & & $\mathrm{H}$ & 3.25085600 & 2.69665100 & 0.86457000 \\
\hline & \multirow{2}{*}{\multicolumn{7}{|c|}{ Cartesian Coordinates }} \\
\hline \multicolumn{7}{|c|}{ Cartesian Coordinates } & \\
\hline 0 & -0.66070400 & -1.69558300 & -0.08895300 & 0 & -0.63639400 & -1.58695400 & -0.05340300 \\
\hline 0 & 2.16329100 & 1.95180000 & 0.07364200 & $\mathrm{O}$ & 2.16046300 & 2.01500400 & -0.03681100 \\
\hline 0 & -0.06683200 & 2.93652700 & 0.45826200 & 0 & -0.26098700 & 3.05288200 & 0.39860900 \\
\hline $\mathrm{O}$ & 4.05766800 & -2.43883000 & -0.33138200 & 0 & 4.00871700 & -2.31256600 & -0.45354200 \\
\hline 0 & -4.46694200 & 1.39836900 & -0.45800300 & 0 & -4.52275900 & 1.28495700 & -0.44314900 \\
\hline 0 & 5.59208700 & -0.31851100 & -0.66186900 & 0 & 5.50852700 & -0.45209100 & -0.56938100 \\
\hline 0 & -4.90631100 & -1.00236500 & -0.73512200 & 0 & -4.83834500 & -1.18278000 & -0.73441500 \\
\hline C & 0.87087000 & 0.00849700 & 0.52755300 & C & 0.84047300 & 0.13296800 & 0.54617500 \\
\hline C & -0.45826100 & 0.59553500 & 0.11058000 & C & -0.50319700 & 0.68921400 & 0.13797200 \\
\hline C & 0.72353400 & -1.39234600 & 0.04933800 & C & 0.68687700 & -1.27762200 & 0.07248900 \\
\hline C & 2.15408900 & 0.65859400 & 0.06573100 & C & 2.11381800 & 0.78266300 & 0.04329500 \\
\hline C & 3.25584700 & -0.16737700 & -0.18978800 & C & 3.23246900 & -0.10312800 & -0.18417600 \\
\hline C & -1.33339100 & -0.43657200 & -0.12540200 & C & -1.33726900 & -0.38094800 & -0.09149500 \\
\hline C & -0.91333900 & 1.91901900 & 0.18621700 & C & -1.03987200 & 1.98703600 & 0.17069300 \\
\hline C & 1.73256700 & -2.18567400 & -0.24576500 & C & 1.68997600 & -2.11183600 & -0.21482400 \\
\hline C & 3.11095900 & -1.62480100 & -0.22683100 & C & 3.02959200 & -1.54407600 & -0.25823900 \\
\hline C & -2.70145200 & -0.25343600 & -0.33457300 & C & -2.71522500 & -0.27989400 & -0.30889000 \\
\hline C & -2.27757200 & 2.16074600 & -0.02106100 & C & -2.41023800 & 2.17798400 & -0.03350600 \\
\hline C & -3.14729500 & 1.10336300 & -0.26955600 & C & -3.22035900 & 1.05736300 & -0.25674800 \\
\hline C & 4.57997400 & 0.38907900 & -0.44442900 & C & 4.54157600 & 0.38514900 & -0.37818400 \\
\hline
\end{tabular}




\begin{tabular}{|c|c|c|c|c|c|c|c|}
\hline C & -3.67339100 & -1.29369300 & -0.58588400 & $\mathrm{C}$ & -3.62310100 & -1.39079600 & -0.58484600 \\
\hline C & -2.83139800 & 3.55758200 & 0.02759100 & C & -3.03063300 & 3.54698100 & -0.02380500 \\
\hline C & 4.76082500 & 1.89486900 & -0.45572400 & C & 4.93062200 & 1.82023900 & -0.37509600 \\
\hline C & -3.25543200 & -2.70781400 & -0.64427600 & C & -3.13146300 & -2.79617100 & -0.69243300 \\
\hline $\mathrm{H}$ & 1.61387500 & -3.20994900 & -0.55445100 & $\mathrm{H}$ & 1.55017700 & -3.14256800 & -0.51013800 \\
\hline $\mathrm{H}$ & -2.01959800 & 4.26883000 & 0.15461400 & $\mathrm{H}$ & -3.64415300 & 3.69463400 & -0.91413500 \\
\hline $\mathrm{H}$ & -3.36896100 & 3.79013100 & -0.89282300 & $\mathrm{H}$ & -3.68173900 & 3.67367300 & 0.84472500 \\
\hline $\mathrm{H}$ & -3.53083900 & 3.66907000 & 0.85832900 & $\mathrm{H}$ & -2.25965400 & 4.31359400 & 0.00652200 \\
\hline $\mathrm{H}$ & 0.96795400 & 2.57890900 & 0.33957400 & $\mathrm{H}$ & 0.68599700 & 2.78670000 & 0.30587100 \\
\hline $\mathrm{H}$ & 5.81460700 & 2.08108800 & -0.65230500 & $\mathrm{H}$ & 6.01475800 & 1.88599500 & -0.43683100 \\
\hline $\mathrm{H}$ & 4.14504200 & 2.35737300 & -1.22455600 & $\mathrm{H}$ & 4.47302700 & 2.32928300 & -1.22510400 \\
\hline $\mathrm{H}$ & 4.46880900 & 2.33224900 & 0.49702000 & $\mathrm{H}$ & 4.56197500 & 2.31591900 & 0.52298900 \\
\hline $\mathrm{H}$ & -4.95085700 & 0.47886200 & -0.61540400 & $\mathrm{H}$ & -4.94746700 & 0.39427200 & -0.59065500 \\
\hline $\mathrm{H}$ & -3.00704800 & -3.03498600 & 0.52877100 & $\mathrm{H}$ & -2.84016500 & -3.14994000 & 0.39554000 \\
\hline $\mathrm{H}$ & -2.32779200 & -2.88098000 & -1.17663300 & $\mathrm{H}$ & -2.23704600 & -2.91358200 & -1.29846600 \\
\hline $\mathrm{H}$ & -4.07245000 & -3.35744300 & -0.94253100 & $\mathrm{H}$ & -3.93924900 & -3.45779100 & -0.99603600 \\
\hline C & 0.92507200 & 0.01608900 & 2.08567400 & $\mathrm{H}$ & 5.05695900 & -1.41754200 & -0.55425100 \\
\hline $\mathrm{H}$ & 1.83653400 & -0.47664300 & 2.42389000 & C & 0.96370400 & 0.12771900 & 2.10290700 \\
\hline $\mathrm{H}$ & 0.91269300 & 1.05644700 & 2.41288000 & $\mathrm{H}$ & 1.88572700 & -0.36731100 & 2.41109600 \\
\hline $\mathrm{H}$ & 0.05001400 & -0.50673300 & 2.47169300 & $\mathrm{H}$ & 0.96162500 & 1.15649900 & 2.46550200 \\
\hline 0 & -2.24383700 & -3.29999100 & 1.51271900 & $\mathrm{H}$ & 0.10932600 & -0.39931800 & 2.53006600 \\
\hline $\mathrm{H}$ & -1.41257500 & -2.84750400 & 1.16120700 & $\mathrm{O}$ & -2.23118300 & -3.50918300 & 1.59319800 \\
\hline & & & & $\mathrm{H}$ & -1.36091800 & -3.11372800 & 1.40672500 \\
\hline \multicolumn{8}{|c|}{ Name: UA-C15-H-OOH-FHT } \\
\hline \multicolumn{8}{|c|}{ Cartesian Coordinates } \\
\hline 0 & -0.74479800 & -1.79489800 & -0.03531700 & 0 & -0.73638500 & -1.75516800 & -0.00827300 \\
\hline 0 & 1.80454300 & 2.01474900 & -0.01985400 & $\mathrm{O}$ & 1.75153800 & 2.06087200 & -0.27837200 \\
\hline 0 & -0.56969300 & 2.84983200 & 0.38976400 & $\mathrm{O}$ & -0.72060400 & 2.91549100 & 0.24890000 \\
\hline 0 & 3.90490100 & -2.19542700 & -0.76589400 & $\mathrm{O}$ & 3.92297700 & -2.12106600 & -0.64534500 \\
\hline $\mathrm{O}$ & -4.79549600 & 0.86977800 & -0.27027400 & 0 & -4.85951100 & 0.76550200 & -0.27012000 \\
\hline $\mathrm{O}$ & 5.36520800 & 0.13782800 & -0.62665400 & $\mathrm{O}$ & 5.25368000 & -0.15249000 & -0.93566800 \\
\hline 0 & -4.98367300 & -1.61768600 & -0.52598000 & 0 & -4.98099400 & -1.72566000 & -0.42904500 \\
\hline C & 0.66807900 & 0.01227400 & 0.48630700 & C & 0.62652700 & 0.10032400 & 0.43254400 \\
\hline C & -0.72547700 & 0.48592200 & 0.14211200 & C & -0.78106700 & 0.53089500 & 0.09069700 \\
\hline C & 0.57395100 & -1.39726300 & 0.01359900 & C & 0.55804600 & -1.34054700 & 0.03059500 \\
\hline C & 1.87084400 & 0.75204000 & -0.07101600 & C & 1.81455400 & 0.83095300 & -0.16641100 \\
\hline C & 3.00562700 & -0.00434800 & -0.44024900 & C & 2.98004700 & 0.03021600 & -0.44312400 \\
\hline C & -1.50717000 & -0.63625000 & -0.03943600 & C & -1.53350200 & -0.61751900 & -0.04053400 \\
\hline C & -1.32091300 & 1.75287300 & 0.19625900 & C & -1.42060500 & 1.78142200 & 0.11045900 \\
\hline C & 1.61498300 & -2.12732200 & -0.37110100 & C & 1.61518100 & -2.09911600 & -0.27370700 \\
\hline C & 2.93080300 & -1.46341200 & -0.49601600 & C & 2.89738700 & -1.42479100 & -0.42656900 \\
\hline C & -2.89833600 & -0.60051400 & -0.19219100 & C & -2.92405000 & -0.63690800 & -0.17090100 \\
\hline C & -2.70864100 & 1.87499000 & 0.04227000 & C & -2.81170500 & 1.84915200 & -0.01560400 \\
\hline C & -3.46263000 & 0.71151100 & -0.13475100 & C & -3.53486500 & 0.65597400 & -0.14771000 \\
\hline C & 4.25864100 & 0.67460100 & -0.78847300 & C & 4.23119800 & 0.61273900 & -0.73255900 \\
\hline C & -3.74922300 & -1.76616900 & -0.40797700 & C & -3.74824600 & -1.83672600 & -0.32844600 \\
\hline C & -3.39602300 & 3.21238800 & 0.06047300 & C & -3.54640300 & 3.16060300 & -0.02292800 \\
\hline C & 4.23447300 & 2.06166300 & -1.38203500 & C & 4.49825300 & 2.07280300 & -0.82095700 \\
\hline C & -3.17595800 & -3.14523400 & -0.49635400 & C & -3.13586900 & -3.20481300 & -0.37173600 \\
\hline $\mathrm{H}$ & 1.53513600 & -3.15659900 & -0.69423600 & $\mathrm{H}$ & 1.55010300 & -3.15085700 & -0.51594300 \\
\hline $\mathrm{H}$ & -4.02167400 & 3.33647500 & -0.82542100 & $\mathrm{H}$ & -4.11751200 & 3.27737300 & -0.94641900 \\
\hline $\mathrm{H}$ & -4.04302700 & 3.30930800 & 0.93568900 & $\mathrm{H}$ & -4.25562500 & 3.21052500 & 0.80620000 \\
\hline $\mathrm{H}$ & -2.66454500 & 4.01704600 & 0.08391100 & $\mathrm{H}$ & -2.84662900 & 3.98854900 & 0.06447400 \\
\hline $\mathrm{H}$ & 0.40912600 & 2.58432300 & 0.29008600 & $\mathrm{H}$ & 0.23897400 & 2.72266600 & 0.11219400 \\
\hline $\mathrm{H}$ & 5.15233500 & 2.19007600 & -1.95545600 & $\mathrm{H}$ & 5.56582400 & 2.22245000 & -0.96755200 \\
\hline $\mathrm{H}$ & 3.36166700 & 2.22963600 & -2.00982600 & $\mathrm{H}$ & 3.93865700 & 2.50322800 & -1.65303200 \\
\hline $\mathrm{H}$ & 4.21703400 & 2.79976000 & -0.57741900 & $\mathrm{H}$ & 4.15690700 & 2.57717100 & 0.08336900 \\
\hline
\end{tabular}




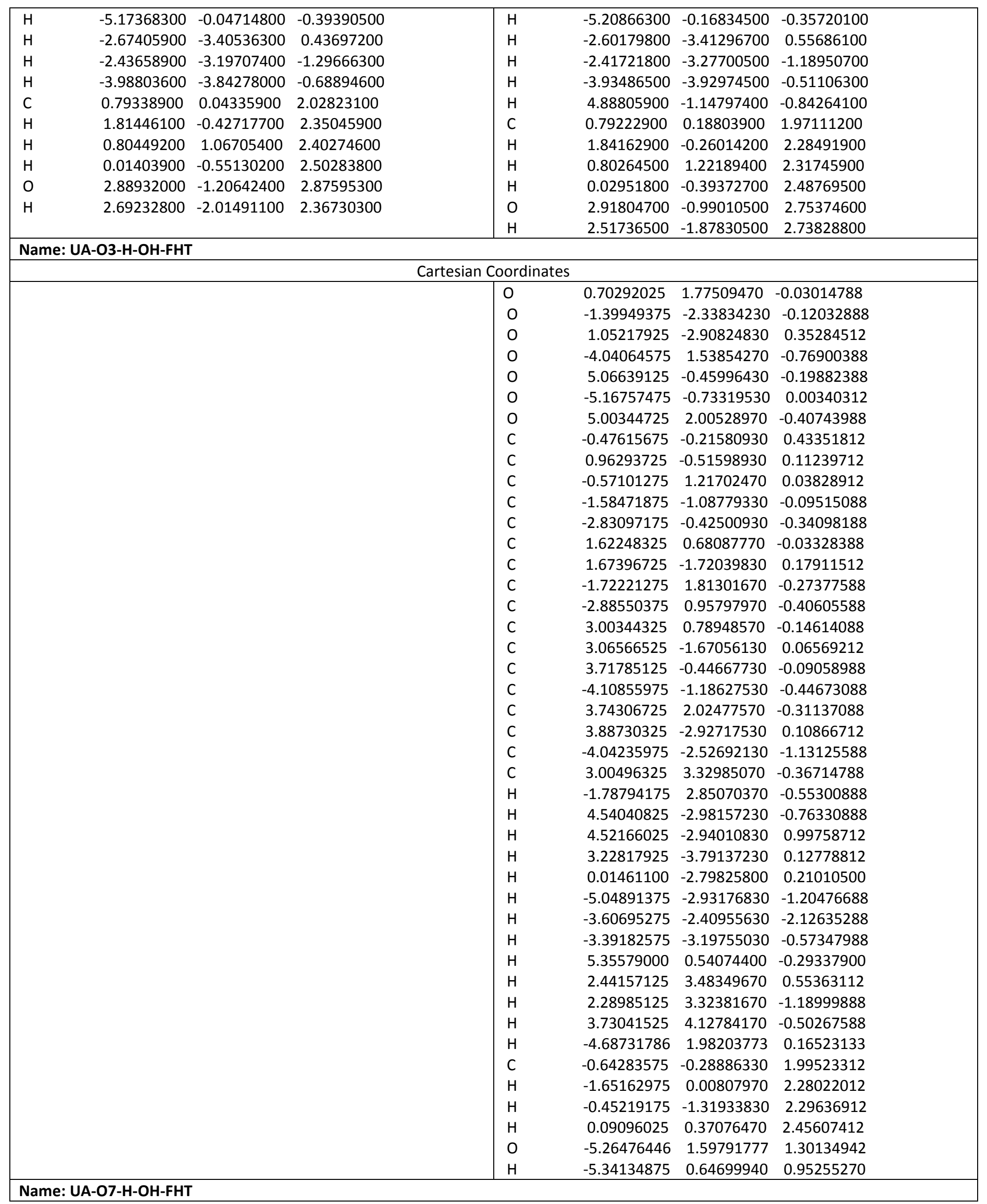




\begin{tabular}{|c|c|c|c|c|c|c|c|}
\hline \multicolumn{8}{|c|}{ Cartesian Coordinates } \\
\hline 0 & 0.33642600 & 1.79631200 & 0.19464900 & 0 & 0.33642600 & 1.79631200 & 0.19464900 \\
\hline $\mathrm{O}$ & -2.20497700 & -1.96711400 & -0.22531000 & 0 & -2.20497700 & -1.96711400 & -0.22531000 \\
\hline 0 & 0.29036300 & -2.88066600 & 0.01950400 & 0 & 0.29036300 & -2.88066600 & 0.01950400 \\
\hline 0 & -4.35104500 & 2.23795800 & -0.03526700 & 0 & -4.35104500 & 2.23795800 & -0.03526700 \\
\hline 0 & 4.39188800 & -0.71981900 & -0.64678800 & 0 & 4.39188800 & -0.71981900 & -0.64678800 \\
\hline 0 & -5.71963400 & 0.31051300 & -0.38507000 & 0 & -5.71963400 & 0.31051300 & -0.38507000 \\
\hline 0 & 4.50355800 & 1.93520600 & -0.32287400 & 0 & 4.50355800 & 1.93520600 & -0.32287400 \\
\hline C & -1.00049400 & -0.08744800 & 0.57816900 & C & -1.00049400 & -0.08744800 & 0.57816900 \\
\hline C & 0.36946600 & -0.49132300 & 0.09228300 & C & 0.36946600 & -0.49132300 & 0.09228300 \\
\hline C & -0.95158200 & 1.38232300 & 0.29917200 & C & -0.95158200 & 1.38232300 & 0.29917200 \\
\hline C & -2.23860900 & -0.75481100 & 0.01386500 & C & -2.23860900 & -0.75481100 & 0.01386500 \\
\hline C & -3.41818900 & 0.07341800 & -0.07709100 & C & -3.41818900 & 0.07341800 & -0.07709100 \\
\hline C & 1.12833600 & 0.67023700 & -0.00057000 & C & 1.12833600 & 0.67023700 & -0.00057000 \\
\hline C & 0.98122100 & -1.73312000 & -0.06234500 & C & 0.98122100 & -1.73312000 & -0.06234500 \\
\hline C & -2.02047700 & 2.17303200 & 0.14935900 & C & -2.02047700 & 2.17303200 & 0.14935900 \\
\hline C & -3.31512500 & 1.52321500 & 0.04324500 & C & -3.31512500 & 1.52321500 & 0.04324500 \\
\hline C & 2.49520800 & 0.70886000 & -0.23043600 & C & 2.49520800 & 0.70886000 & -0.23043600 \\
\hline C & 2.36757900 & -1.78424800 & -0.28839000 & C & 2.36757900 & -1.78424800 & -0.28839000 \\
\hline C & 3.09613200 & -0.58298000 & -0.36100500 & C & 3.09613200 & -0.58298000 & -0.36100500 \\
\hline C & -4.69465700 & -0.47505800 & -0.32141500 & C & -4.69465700 & -0.47505800 & -0.32141500 \\
\hline C & 3.28403200 & 1.96133900 & -0.39013800 & C & 3.28403200 & 1.96133900 & -0.39013800 \\
\hline C & 3.07935900 & -3.09675300 & -0.44439500 & C & 3.07935900 & -3.09675300 & -0.44439500 \\
\hline C & -4.98518200 & -1.92142200 & -0.51066000 & C & -4.98518200 & -1.92142200 & -0.51066000 \\
\hline C & 2.57212800 & 3.26215800 & -0.65818000 & C & 2.57212800 & 3.26215800 & -0.65818000 \\
\hline $\mathrm{H}$ & -1.95936100 & 3.24210900 & 0.00130400 & $\mathrm{H}$ & -1.95936100 & 3.24210900 & 0.00130400 \\
\hline $\mathrm{H}$ & 3.90896500 & -3.16536100 & 0.26228900 & $\mathrm{H}$ & 3.90896500 & -3.16536100 & 0.26228900 \\
\hline $\mathrm{H}$ & 2.39458500 & -3.92307600 & -0.27031600 & $\mathrm{H}$ & 2.39458500 & -3.92307600 & -0.27031600 \\
\hline $\mathrm{H}$ & 3.49895900 & -3.19365700 & -1.44811300 & $\mathrm{H}$ & 3.49895900 & -3.19365700 & -1.44811300 \\
\hline $\mathrm{H}$ & -0.67539700 & -2.67678200 & -0.02151800 & $\mathrm{H}$ & -0.67539700 & -2.67678200 & -0.02151800 \\
\hline $\mathrm{H}$ & -6.06287200 & -2.05253700 & -0.58208500 & $\mathrm{H}$ & -6.06287200 & -2.05253700 & -0.58208500 \\
\hline $\mathrm{H}$ & -4.49949700 & -2.28106900 & -1.41922600 & $\mathrm{H}$ & -4.49949700 & -2.28106900 & -1.41922600 \\
\hline $\mathrm{H}$ & -4.57731700 & -2.50441000 & 0.31530300 & $\mathrm{H}$ & -4.57731700 & -2.50441000 & 0.31530300 \\
\hline $\mathrm{H}$ & 5.04881900 & -0.12037400 & -0.04166700 & $\mathrm{H}$ & 5.04881900 & -0.12037400 & -0.04166700 \\
\hline $\mathrm{H}$ & 2.07158000 & 3.60783800 & 0.24836900 & $\mathrm{H}$ & 2.07158000 & 3.60783800 & 0.24836900 \\
\hline $\mathrm{H}$ & 1.81554000 & 3.15231500 & -1.43576300 & $\mathrm{H}$ & 1.81554000 & 3.15231500 & -1.43576300 \\
\hline $\mathrm{H}$ & 3.32065900 & 3.99328000 & -0.95747900 & $\mathrm{H}$ & 3.32065900 & 3.99328000 & -0.95747900 \\
\hline C & -1.08109300 & -0.30068800 & 2.12474900 & $\mathrm{H}$ & -5.33148900 & 1.29634700 & -0.24242900 \\
\hline $\mathrm{H}$ & -2.02827200 & 0.07956700 & 2.51052000 & C & -1.08109300 & -0.30068800 & 2.12474900 \\
\hline $\mathrm{H}$ & -0.99699000 & -1.36617600 & 2.34266900 & $\mathrm{H}$ & -2.02827200 & 0.07956700 & 2.51052000 \\
\hline $\mathrm{H}$ & -0.25619200 & 0.22696500 & 2.60495800 & $\mathrm{H}$ & -0.99699000 & -1.36617600 & 2.34266900 \\
\hline 0 & 5.76472300 & -0.25812700 & 1.07430900 & $\mathrm{H}$ & -0.25619200 & 0.22696500 & 2.60495800 \\
\hline $\mathrm{H}$ & 6.22955200 & -1.10947200 & 1.01064200 & 0 & 5.76472300 & -0.25812700 & 1.07430900 \\
\hline & & & & $\mathrm{H}$ & 6.22955200 & -1.10947200 & 1.01064200 \\
\hline \multicolumn{8}{|c|}{ Name: UA-O9-H-OH-FHT } \\
\hline \multicolumn{8}{|c|}{ Cartesian Coordinates } \\
\hline 0 & -0.68128700 & -1.88468300 & 0.29645000 & 0 & -0.68128700 & -1.88468300 & 0.29645000 \\
\hline $\mathrm{O}$ & 1.93841400 & 1.68086100 & -0.66058100 & $\mathrm{O}$ & 1.93841400 & 1.68086100 & -0.66058100 \\
\hline $\mathrm{O}$ & -0.66197400 & 2.79067800 & -0.23866000 & 0 & -0.66197400 & 2.79067800 & -0.23866000 \\
\hline $\mathrm{O}$ & 3.97324700 & -2.53570900 & -0.07057400 & 0 & 3.97324700 & -2.53570900 & -0.07057400 \\
\hline 0 & -4.75311900 & 0.58103200 & -0.50816200 & 0 & -4.75311900 & 0.58103200 & -0.50816200 \\
\hline 0 & 5.41385900 & -0.67384300 & -0.53013800 & 0 & 5.41385900 & -0.67384300 & -0.53013800 \\
\hline 0 & -4.87410000 & -1.90485400 & -0.28013900 & 0 & -4.87410000 & -1.90485400 & -0.28013900 \\
\hline C & 0.73409400 & -0.02384400 & 0.49215300 & C & 0.73409400 & -0.02384400 & 0.49215300 \\
\hline C & -0.66345800 & 0.39715900 & 0.08908000 & C & -0.66345800 & 0.39715900 & 0.08908000 \\
\hline C & 0.62453400 & -1.50753000 & 0.30641300 & C & 0.62453400 & -1.50753000 & 0.30641300 \\
\hline C & 1.95352300 & 0.53889900 & -0.22832700 & C & 1.95352300 & 0.53889900 & -0.22832700 \\
\hline
\end{tabular}




\begin{tabular}{|c|c|c|c|c|c|c|c|}
\hline C & 3.11995200 & -0.34251000 & -0.24277400 & C & 3.11995200 & -0.34251000 & -0.24277400 \\
\hline C & -1.43872600 & -0.74342700 & 0.09462100 & c & -1.43872600 & -0.74342700 & 0.09462100 \\
\hline C & -1.30938000 & 1.63670900 & -0.12252300 & c & -1.30938000 & 1.63670900 & -0.12252300 \\
\hline C & 1.65032600 & -2.35290200 & 0.15999300 & C & 1.65032600 & -2.35290200 & 0.15999300 \\
\hline C & 2.97053800 & -1.77158500 & -0.02345500 & C & 2.97053800 & -1.77158500 & -0.02345500 \\
\hline C & -2.82735700 & -0.78227100 & -0.09580400 & C & -2.82735700 & -0.78227100 & -0.09580400 \\
\hline C & -2.70608000 & 1.68209800 & -0.30789500 & C & -2.70608000 & 1.68209800 & -0.30789500 \\
\hline C & -3.43734200 & 0.48413600 & -0.30482700 & C & -3.43734200 & 0.48413600 & -0.30482700 \\
\hline C & 4.40608900 & 0.14633800 & -0.51603100 & C & 4.40608900 & 0.14633800 & -0.51603100 \\
\hline C & -3.65147400 & -2.00207000 & -0.10083300 & C & -3.65147400 & -2.00207000 & -0.10083300 \\
\hline C & -3.43366700 & 2.97387600 & -0.53602300 & C & -3.43366700 & 2.97387600 & -0.53602300 \\
\hline C & 4.74771600 & 1.56962900 & -0.78733500 & C & 4.74771600 & 1.56962900 & -0.78733500 \\
\hline C & -3.05224100 & -3.35945900 & 0.10531600 & C & -3.05224100 & -3.35945900 & 0.10531600 \\
\hline $\mathrm{H}$ & 1.53385700 & -3.42552400 & 0.09005800 & $\mathrm{H}$ & 1.53385700 & -3.42552400 & 0.09005800 \\
\hline $\mathrm{H}$ & -4.31002400 & 3.03110500 & 0.11147500 & $\mathrm{H}$ & -4.31002400 & 3.03110500 & 0.11147500 \\
\hline $\mathrm{H}$ & -2.78268500 & 3.82179500 & -0.33986300 & $\mathrm{H}$ & -2.78268500 & 3.82179500 & -0.33986300 \\
\hline $\mathrm{H}$ & -3.78951600 & 3.03891100 & -1.56794500 & $\mathrm{H}$ & -3.78951600 & 3.03891100 & -1.56794500 \\
\hline $\mathrm{H}$ & 0.24842100 & 2.91291900 & 0.31557100 & $\mathrm{H}$ & 0.24842100 & 2.91291900 & 0.31557100 \\
\hline $\mathrm{H}$ & 5.82949600 & 1.68051700 & -0.74243700 & $\mathrm{H}$ & 5.82949600 & 1.68051700 & -0.74243700 \\
\hline $\mathrm{H}$ & 4.38876200 & 1.84744900 & -1.78031200 & $\mathrm{H}$ & 4.38876200 & 1.84744900 & -1.78031200 \\
\hline $\mathrm{H}$ & 4.25564200 & 2.23231000 & -0.07675700 & $\mathrm{H}$ & 4.25564200 & 2.23231000 & -0.07675700 \\
\hline $\mathrm{H}$ & -5.10995200 & -0.35063700 & -0.46691300 & $\mathrm{H}$ & -5.10995200 & -0.35063700 & -0.46691300 \\
\hline $\mathrm{H}$ & -2.53433400 & -3.40895600 & 1.06420200 & $\mathrm{H}$ & -2.53433400 & -3.40895600 & 1.06420200 \\
\hline $\mathrm{H}$ & -2.32047600 & -3.57419900 & -0.67511100 & $\mathrm{H}$ & -2.32047600 & -3.57419900 & -0.67511100 \\
\hline $\mathrm{H}$ & -3.85670900 & -4.09100000 & 0.07499700 & $\mathrm{H}$ & -3.85670900 & -4.09100000 & 0.07499700 \\
\hline C & 0.96197700 & 0.24354000 & 2.01474800 & $\mathrm{H}$ & 4.99956400 & -1.62997700 & -0.33793500 \\
\hline $\mathrm{H}$ & 1.91147900 & -0.19089900 & 2.33077800 & C & 0.96197700 & 0.24354000 & 2.01474800 \\
\hline $\mathrm{H}$ & 0.96678500 & 1.31650900 & 2.19715900 & $\mathrm{H}$ & 1.91147900 & -0.19089900 & 2.33077800 \\
\hline $\mathrm{H}$ & 0.15170100 & -0.21507800 & 2.58397400 & $\mathrm{H}$ & 0.96678500 & 1.31650900 & 2.19715900 \\
\hline 0 & 0.67280200 & 3.43990600 & 1.45862000 & $\mathrm{H}$ & 0.15170100 & -0.21507800 & 2.58397400 \\
\hline $\mathrm{H}$ & 0.30111300 & 4.33693800 & 1.50554700 & 0 & 0.67280200 & 3.43990600 & 1.45862000 \\
\hline & & & & $\mathrm{H}$ & 0.30111300 & 4.33693800 & 1.50554700 \\
\hline \multicolumn{8}{|c|}{ Name: UA-C2-OH-RAF } \\
\hline \multicolumn{8}{|c|}{ Cartesian Coordinates } \\
\hline 0 & -0.79174600 & -1.80180500 & 0.25848300 & 0 & -0.78643700 & -1.77836700 & 0.25929900 \\
\hline 0 & 1.89828600 & 1.94243700 & 0.08199000 & $\mathrm{O}$ & 1.83666900 & 1.96000700 & -0.01167400 \\
\hline $\mathrm{O}$ & -0.54290400 & 2.86044700 & 0.30443200 & 0 & -0.70459700 & 2.90137400 & 0.27861200 \\
\hline 0 & 3.91550800 & -2.31777800 & 0.02569200 & 0 & 3.90809800 & -2.30696600 & 0.12609300 \\
\hline $\mathrm{O}$ & -4.77203100 & 0.90350400 & -0.40526600 & 0 & -4.83861700 & 0.78903600 & -0.39622100 \\
\hline 0 & 4.96087700 & 0.23505700 & 0.93067000 & 0 & 5.33125400 & -0.34744000 & -0.11815300 \\
\hline 0 & -5.00864300 & -1.59161500 & -0.42233500 & 0 & -4.99695500 & -1.70724700 & -0.42089400 \\
\hline C & 0.62425000 & 0.02202600 & 0.69507300 & C & 0.58226400 & 0.07658400 & 0.67263900 \\
\hline C & -0.73804700 & 0.48369700 & 0.24489100 & C & -0.79268200 & 0.51001900 & 0.22857500 \\
\hline C & 0.52526100 & -1.42934000 & 0.35590300 & C & 0.50432300 & -1.38360500 & 0.37354100 \\
\hline C & 1.85681200 & 0.67585300 & 0.12057300 & C & 1.81385500 & 0.74207300 & 0.10187500 \\
\hline C & 2.96009200 & -0.15880400 & -0.23583000 & C & 2.98608200 & -0.12694300 & -0.17803300 \\
\hline C & -1.53281200 & -0.63355800 & 0.11862900 & C & -1.55816500 & -0.63000800 & 0.11270900 \\
\hline C & -1.30685100 & 1.75917500 & 0.16487300 & C & -1.41108800 & 1.76692600 & 0.14812500 \\
\hline C & 1.56622400 & -2.23881700 & 0.14405500 & C & 1.57714200 & -2.17812000 & 0.22747400 \\
\hline C & 2.89145100 & -1.62047900 & 0.03971000 & C & 2.85118300 & -1.52728700 & 0.12297300 \\
\hline C & -2.91507600 & -0.58848900 & -0.10094900 & C & -2.93942700 & -0.63633400 & -0.09985700 \\
\hline C & -2.68183300 & 1.89417600 & -0.06651400 & C & -2.78958200 & 1.85080500 & -0.06885700 \\
\hline C & -3.45140900 & 0.73265400 & -0.18645100 & C & -3.52602200 & 0.66389400 & -0.18496300 \\
\hline C & 4.31279400 & 0.48966600 & -0.07764800 & C & 4.37215000 & 0.43394900 & -0.19417400 \\
\hline C & -3.78509400 & -1.75295500 & -0.23275200 & C & -3.77490600 & -1.83074300 & -0.23935400 \\
\hline C & -3.34352400 & 3.23857800 & -0.18720400 & C & -3.49783100 & 3.17098900 & -0.19078200 \\
\hline C & 4.82593500 & 1.43389500 & -1.11543900 & C & 4.62084300 & 1.89654400 & -0.33246000 \\
\hline
\end{tabular}




\begin{tabular}{|c|c|c|c|c|c|c|c|}
\hline C & -3.24463800 & -3.14512300 & -0.14081100 & C & -3.18656600 & -3.20829700 & -0.16765900 \\
\hline $\mathrm{H}$ & 1.46958700 & -3.30069100 & -0.03858100 & $\mathrm{H}$ & 1.51847400 & -3.25115100 & 0.10606000 \\
\hline $\mathrm{H}$ & -4.04570300 & 3.40383900 & 0.63344900 & $\mathrm{H}$ & -2.79276900 & 3.99246400 & -0.08722200 \\
\hline $\mathrm{H}$ & -2.60114700 & 4.03329500 & -0.16854200 & $\mathrm{H}$ & -3.99428500 & 3.25282800 & -1.16013000 \\
\hline $\mathrm{H}$ & -3.90624900 & 3.30946800 & -1.11997200 & $\mathrm{H}$ & -4.26782000 & 3.26816700 & 0.57760500 \\
\hline $\mathrm{H}$ & 0.42472200 & 2.59188800 & 0.28643000 & $\mathrm{H}$ & 0.25457900 & 2.69976500 & 0.23028300 \\
\hline $\mathrm{H}$ & 5.21083200 & 0.83874200 & -1.94793700 & $\mathrm{H}$ & 5.69156300 & 2.04727300 & -0.45355400 \\
\hline $\mathrm{H}$ & 4.02699400 & 2.06245300 & -1.50420600 & $\mathrm{H}$ & 4.06898800 & 2.29139100 & -1.18378300 \\
\hline $\mathrm{H}$ & 5.63041600 & 2.03132000 & -0.69022100 & $\mathrm{H}$ & 4.26543200 & 2.41840300 & 0.55827400 \\
\hline $\mathrm{H}$ & -5.16546300 & -0.01375000 & -0.46131100 & $\mathrm{H}$ & -5.20130900 & -0.14107700 & -0.45215000 \\
\hline $\mathrm{H}$ & -2.77953900 & -3.30372900 & 0.83345000 & $\mathrm{H}$ & -2.71634300 & -3.37145700 & 0.80354700 \\
\hline $\mathrm{H}$ & -2.48179200 & -3.30781700 & -0.90333800 & $\mathrm{H}$ & -2.41937000 & -3.33755800 & -0.93250800 \\
\hline $\mathrm{H}$ & -4.06696300 & -3.84344200 & -0.27923000 & $\mathrm{H}$ & -3.98864000 & -3.92774000 & -0.31735700 \\
\hline C & 0.73721600 & 0.16898400 & 2.24279700 & $\mathrm{H}$ & 4.71311500 & -1.67837300 & 0.02072700 \\
\hline $\mathrm{H}$ & 1.68445300 & -0.25166400 & 2.58528800 & C & 0.72834700 & 0.25373300 & 2.22140200 \\
\hline $\mathrm{H}$ & 0.69137600 & 1.22797200 & 2.49971000 & $\mathrm{H}$ & 1.68773200 & -0.14066700 & 2.56210400 \\
\hline $\mathrm{H}$ & -0.09094300 & -0.35507400 & 2.72185400 & $\mathrm{H}$ & 0.66098400 & 1.31436800 & 2.46615200 \\
\hline 0 & 2.86906000 & -0.28962400 & -2.13918100 & $\mathrm{H}$ & -0.08063800 & -0.28159800 & 2.71925600 \\
\hline $\mathrm{H}$ & 1.96765400 & -0.63461800 & -2.22013800 & $\mathrm{O}$ & 2.79832600 & 0.06863800 & -2.02362300 \\
\hline & & & & $\mathrm{H}$ & 2.85886400 & -0.85238700 & -2.32384600 \\
\hline \multicolumn{8}{|c|}{ Name: UA-C3-OH-RAF } \\
\hline \multicolumn{8}{|c|}{ Cartesian Coordinates } \\
\hline & & & & 0 & -0.72966200 & -1.74297100 & 0.08095800 \\
\hline & & & & 0 & 1.63116700 & 2.12074200 & -0.22755600 \\
\hline & & & & $\mathrm{O}$ & -0.84343900 & 2.92421600 & 0.32179300 \\
\hline & & & & $\mathrm{O}$ & 3.93951600 & -2.02959800 & -0.59694500 \\
\hline & & & & $\mathrm{O}$ & -4.91049400 & 0.66373000 & -0.30484900 \\
\hline & & & & $\mathrm{O}$ & 5.22943600 & 0.07967600 & -0.84409900 \\
\hline & & & & $\mathrm{O}$ & -4.95614900 & -1.82893600 & -0.47112400 \\
\hline & & & & C & 0.57426900 & 0.15357300 & 0.56478000 \\
\hline & & & & C & -0.83445200 & 0.53955500 & 0.17606000 \\
\hline & & & & C & 0.55507100 & -1.29248000 & 0.17053100 \\
\hline & & & & C & 1.74077500 & 0.89985600 & -0.05566700 \\
\hline & & & & C & 2.94376900 & 0.13986400 & -0.29922900 \\
\hline & & & & C & -1.55103200 & -0.62662400 & 0.01916500 \\
\hline & & & & C & -1.50982600 & 1.77035800 & 0.17095600 \\
\hline & & & & C & 1.63342800 & -2.02622800 & -0.09870500 \\
\hline & & & & C & 2.92808500 & -1.33581100 & -0.13928900 \\
\hline & & & & C & -2.93817400 & -0.68323400 & -0.15322500 \\
\hline & & & & C & -2.89922000 & 1.80223200 & 0.00695700 \\
\hline & & & & C & -3.58577800 & 0.59104200 & -0.14528800 \\
\hline & & & & C & 4.13707700 & 0.77303800 & -0.64170600 \\
\hline & & & & C & -3.72280300 & -1.90338300 & -0.33705200 \\
\hline & & & & C & -3.66748200 & 3.09414100 & -0.02310700 \\
\hline & & & & C & 4.32368800 & 2.23986400 & -0.81181500 \\
\hline & & & & C & -3.07182400 & -3.25428200 & -0.36897200 \\
\hline & & & & $\mathrm{H}$ & 1.60241900 & -3.07549800 & -0.35984600 \\
\hline & & & & $\mathrm{H}$ & -2.99194800 & 3.93994800 & 0.08236300 \\
\hline & & & & $\mathrm{H}$ & -4.21548300 & 3.19546500 & -0.96238600 \\
\hline & & & & $\mathrm{H}$ & -4.40100600 & 3.12714600 & 0.78560700 \\
\hline & & & & $\mathrm{H}$ & 0.12059400 & 2.75339300 & 0.18162000 \\
\hline & & & & $\mathrm{H}$ & 5.37705000 & 2.43306700 & -1.00363900 \\
\hline & & & & $\mathrm{H}$ & 3.71739100 & 2.60075900 & -1.64375100 \\
\hline & & & & $\mathrm{H}$ & 3.98920100 & 2.77474900 & 0.07701500 \\
\hline & & & & $\mathrm{H}$ & -5.22859400 & -0.28016400 & -0.40322800 \\
\hline & & & & $\mathrm{H}$ & -2.56802900 & -3.45628000 & 0.57777200 \\
\hline & & & & $\mathrm{H}$ & -2.31993000 & -3.29949900 & -1.15818400 \\
\hline
\end{tabular}




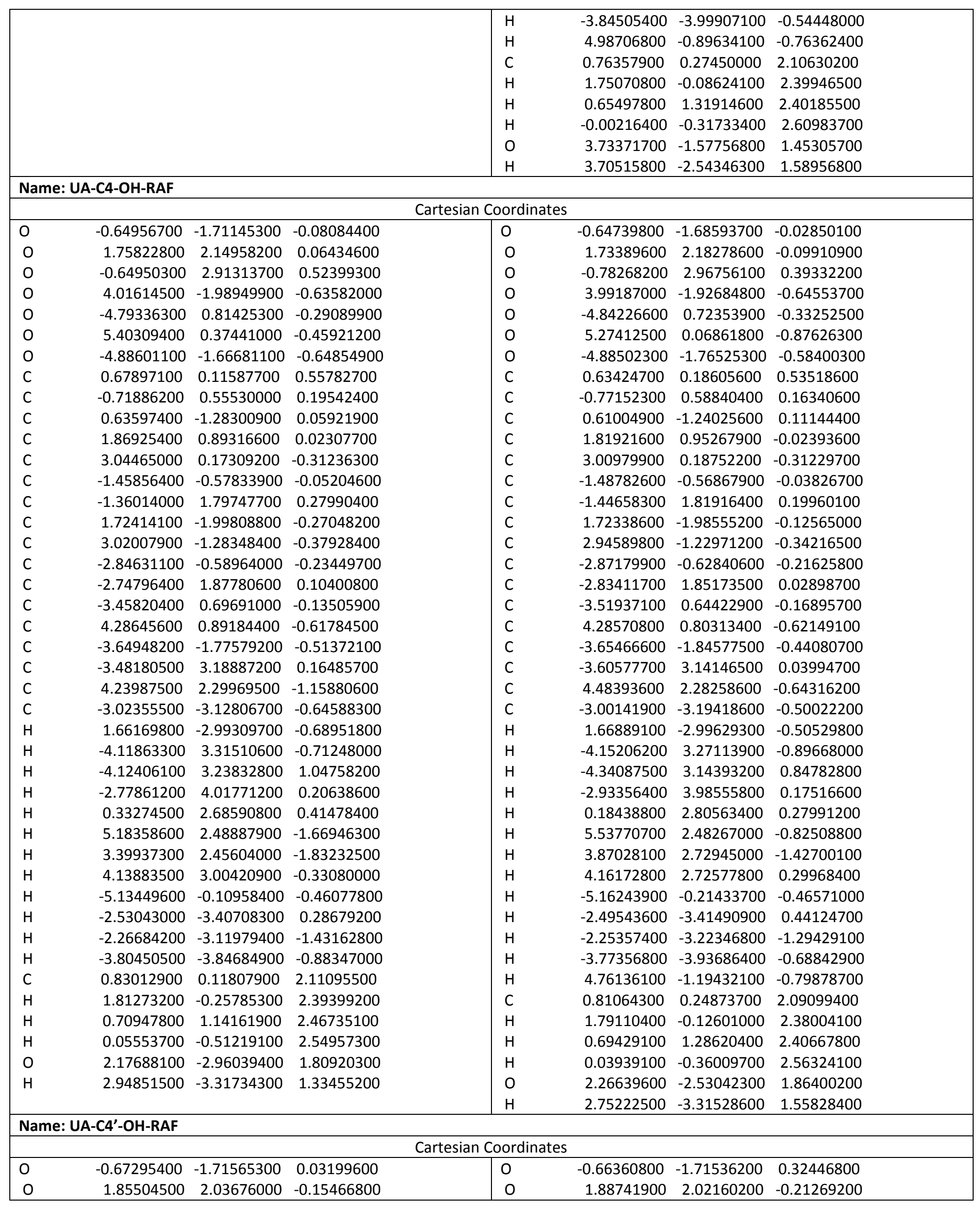




\begin{tabular}{|c|c|c|c|c|c|c|c|}
\hline 0 & -0.50020600 & 2.93847800 & 0.33959300 & 0 & -0.60882600 & 2.95731100 & 0.13425700 \\
\hline $\mathrm{O}$ & 3.97792900 & -2.19899400 & -0.53179000 & $\mathrm{O}$ & 4.03322300 & -2.18489000 & 0.04980000 \\
\hline 0 & -4.70810400 & 0.94733800 & -0.39264000 & 0 & -4.76047400 & 0.80621100 & -0.30779900 \\
\hline 0 & 5.25685200 & 0.12221600 & -1.29846800 & 0 & 5.41225100 & -0.27061200 & -0.30019100 \\
\hline 0 & -4.88824400 & -1.54685200 & -0.60725200 & 0 & -4.90067400 & -1.69508600 & -0.24467300 \\
\hline C & 0.72960000 & 0.10305700 & 0.59059000 & C & 0.69044600 & 0.15831300 & 0.63043800 \\
\hline C & -0.65080800 & 0.56396800 & 0.17417500 & C & -0.69808500 & 0.57166000 & 0.21711400 \\
\hline C & 0.62699600 & -1.33932000 & 0.19644200 & C & 0.61511800 & -1.29622500 & 0.31277900 \\
\hline C & 1.93565300 & 0.78595000 & -0.03702500 & C & 1.92236200 & 0.81447500 & 0.04389900 \\
\hline C & 3.09086700 & 0.01649800 & -0.33969500 & C & 3.10695200 & -0.01628500 & -0.02153700 \\
\hline C & -1.43193800 & -0.55197200 & -0.01678700 & C & -1.45856900 & -0.57349100 & 0.16167500 \\
\hline C & -1.24686700 & 1.83234600 & 0.16628800 & C & -1.32076900 & 1.82145500 & 0.08870600 \\
\hline C & 1.67387400 & -2.06537800 & -0.27375000 & C & 1.71493900 & -2.10499100 & 0.23567400 \\
\hline C & 3.00768400 & -1.43306000 & -0.367777700 & C & 2.98359600 & -1.42536300 & 0.10976800 \\
\hline C & -2.81603600 & -0.52241700 & -0.22289800 & c & -2.84413500 & -0.59877000 & -0.01308500 \\
\hline C & -2.62857300 & 1.95365600 & -0.03218100 & C & -2.70683600 & 1.88889000 & -0.08942300 \\
\hline C & -3.38073200 & 0.78873300 & -0.20921500 & C & -3.44019700 & 0.69537000 & -0.13146600 \\
\hline C & 4.35824700 & 0.68551100 & -0.65514800 & C & 4.42942800 & 0.51519400 & -0.27024400 \\
\hline C & -3.65852100 & -1.69302500 & -0.44977700 & C & -3.67347400 & -1.80199700 & -0.09221700 \\
\hline C & -3.31216000 & 3.29246700 & -0.06993200 & C & -3.42448500 & 3.19942900 & -0.25097000 \\
\hline C & 4.62511200 & 2.08885100 & -0.16787400 & C & 4.68544800 & 1.97047100 & -0.48651800 \\
\hline C & -3.08147300 & -3.07259000 & -0.50455200 & C & -3.06986200 & -3.17188600 & 0.00508100 \\
\hline $\mathrm{H}$ & 1.55457600 & -3.06678400 & -0.66471200 & $\mathrm{H}$ & 1.67199800 & -3.18270800 & 0.17046200 \\
\hline $\mathrm{H}$ & -2.57993300 & 4.09624300 & -0.04353300 & $\mathrm{H}$ & -3.97651300 & 3.22190500 & -1.19283200 \\
\hline $\mathrm{H}$ & -3.90951400 & 3.39472000 & -0.97783500 & $\mathrm{H}$ & -4.14885200 & 3.34529200 & 0.55347400 \\
\hline H & -3.98588700 & 3.41199500 & 0.78198400 & $\mathrm{H}$ & -2.71631400 & 4.02467100 & -0.23835500 \\
\hline $\mathrm{H}$ & 0.46821100 & 2.67497900 & 0.21888500 & $\mathrm{H}$ & 0.34990500 & 2.74060300 & 0.04224500 \\
\hline$H$ & 5.70547000 & 2.20557200 & -0.08504700 & $\mathrm{H}$ & 4.17528300 & 2.30715700 & -1.39026700 \\
\hline $\mathrm{H}$ & 4.25145100 & 2.80700500 & -0.90022400 & $\mathrm{H}$ & 4.28334400 & 2.55809400 & 0.33944300 \\
\hline $\mathrm{H}$ & 4.14237500 & 2.29793200 & 0.78486500 & $\mathrm{H}$ & 5.75938400 & 2.11798300 & -0.57901600 \\
\hline $\mathrm{H}$ & -5.08419700 & 0.02981100 & -0.51162100 & $\mathrm{H}$ & -5.11926600 & -0.12540300 & -0.32147800 \\
\hline H & -2.61400600 & -3.32285900 & 0.44927200 & $\mathrm{H}$ & -2.56478500 & -3.29947000 & 0.96387900 \\
\hline H & -2.31334200 & -3.13152000 & -1.27683100 & $\mathrm{H}$ & -2.32905000 & -3.31942500 & -0.78245800 \\
\hline $\mathrm{H}$ & -3.88580400 & -3.77249900 & -0.71956600 & $\mathrm{H}$ & -3.86928400 & -3.90288300 & -0.09388000 \\
\hline C & 0.90038700 & 0.30675800 & 2.12270700 & $\mathrm{H}$ & 4.84244700 & -1.49965100 & -0.10634900 \\
\hline $\mathrm{H}$ & 1.85744500 & -0.09334100 & 2.45437400 & C & 0.83201700 & 0.30952200 & 2.18073400 \\
\hline $\mathrm{H}$ & 0.85883900 & 1.37700900 & 2.32761900 & $\mathrm{H}$ & 1.78450900 & -0.09399600 & 2.52619000 \\
\hline $\mathrm{H}$ & 0.09058100 & -0.19715700 & 2.64752500 & $\mathrm{H}$ & 0.77266300 & 1.36881500 & 2.43411600 \\
\hline 0 & 1.19521600 & -2.55213900 & 1.92722700 & $\mathrm{H}$ & 0.01343700 & -0.21660600 & 2.67299200 \\
\hline $\mathrm{H}$ & 0.87159100 & -3.40053500 & 1.58130600 & 0 & 0.91978900 & -1.14702000 & -1.78326900 \\
\hline & & & & $\mathrm{H}$ & 0.45494200 & -1.95625600 & -2.05983100 \\
\hline \multicolumn{8}{|c|}{-C6-OH-RAF } \\
\hline \multicolumn{8}{|c|}{ Cartesian Coordinates } \\
\hline 0 & -0.53892400 & -1.77262500 & 0.18363800 & 0 & -0.53417800 & -1.72556800 & -0.01641000 \\
\hline 0 & 2.15133500 & 1.91194300 & -0.02188100 & $\mathrm{O}$ & 2.13505600 & 1.97485500 & -0.12537100 \\
\hline $\mathrm{O}$ & -0.16836100 & 2.87067200 & 0.23125800 & 0 & -0.33338700 & 2.93667900 & 0.16132300 \\
\hline 0 & 4.13681900 & -2.42265100 & -0.07780400 & 0 & 4.13881300 & -2.29477500 & -0.28252700 \\
\hline 0 & -4.45824200 & 1.03874800 & -0.43621700 & 0 & -4.52182700 & 0.95775000 & -0.60319500 \\
\hline $\mathrm{O}$ & 5.67375000 & -0.16874300 & -0.29729000 & 0 & 5.56064400 & -0.43466400 & -0.47118000 \\
\hline $\mathrm{O}$ & -4.62956800 & -1.41854000 & -1.02773600 & 0 & -4.73741300 & -1.52622300 & -0.73797300 \\
\hline C & 0.92157600 & 0.01317400 & 0.63582500 & C & 0.86351800 & 0.07736400 & 0.50450300 \\
\hline C & -0.42627100 & 0.50941000 & 0.17458100 & C & -0.48672000 & 0.55853800 & 0.03476300 \\
\hline C & 0.79218300 & -1.42851800 & 0.28757100 & C & 0.76587800 & -1.36530200 & 0.12556700 \\
\hline C & 2.17763800 & 0.64916800 & 0.07735000 & C & 2.12879600 & 0.74435800 & 0.00854600 \\
\hline C & 3.30640300 & -0.17725300 & -0.12839000 & C & 3.27682100 & -0.11259400 & -0.14339700 \\
\hline C & -1.23486600 & -0.58308800 & 0.03280000 & C & -1.27459000 & -0.55758600 & -0.12768300 \\
\hline C & -0.96374700 & 1.80836600 & 0.10677700 & C & -1.07193000 & 1.83363900 & -0.00741500 \\
\hline
\end{tabular}




\begin{tabular}{|c|c|c|c|c|c|c|c|}
\hline C & 1.82439200 & -2.23310700 & 0.05983900 & C & 1.81335800 & -2.17572100 & -0.07605100 \\
\hline C & 3.17454600 & -1.63201800 & -0.00909700 & C & 3.11358700 & -1.54480500 & -0.13866100 \\
\hline C & -2.65620300 & -0.52238300 & -0.08827300 & C & -2.65945100 & -0.52231500 & -0.33915400 \\
\hline C & -2.34684400 & 1.97577300 & -0.10665800 & C & -2.44936200 & 1.96069200 & -0.22929100 \\
\hline C & -3.14173300 & 0.84751800 & -0.22906600 & C & -3.21335100 & 0.79866400 & -0.38931400 \\
\hline C & 4.59539100 & 0.41116300 & -0.50143800 & C & 4.59645900 & 0.39147000 & -0.34954200 \\
\hline C & -3.46151400 & -1.63520300 & -0.69007300 & C & -3.52449000 & -1.69239200 & -0.53801800 \\
\hline C & -2.96803100 & 3.33896600 & -0.23330500 & C & -3.12091500 & 3.30270600 & -0.31700900 \\
\hline C & 4.65151900 & 1.75770400 & -1.18109600 & C & 4.93186800 & 1.84091800 & -0.42519200 \\
\hline C & -2.86737700 & -2.99343900 & -0.86257700 & C & -2.96849600 & -3.08258600 & -0.50836400 \\
\hline $\mathrm{H}$ & 1.72852200 & -3.28860600 & -0.15625400 & $\mathrm{H}$ & 1.72730600 & -3.23012600 & -0.29725700 \\
\hline $\mathrm{H}$ & -2.20241500 & 4.11108700 & -0.21066500 & $\mathrm{H}$ & -3.62365800 & 3.41850400 & -1.27951500 \\
\hline H & -3.52165600 & 3.42402700 & -1.17020200 & $\mathrm{H}$ & -3.88094000 & 3.40790800 & 0.46035000 \\
\hline $\mathrm{H}$ & -3.67021600 & 3.52400400 & 0.58275000 & $\mathrm{H}$ & -2.39129000 & 4.10110300 & -0.20322700 \\
\hline $\mathrm{H}$ & 0.80981700 & 2.54533200 & 0.18160800 & $\mathrm{H}$ & 0.62590200 & 2.69580500 & 0.10978700 \\
\hline $\mathrm{H}$ & 5.60382300 & 1.82056100 & -1.70657900 & $\mathrm{H}$ & 6.01265000 & 1.94220400 & -0.49841300 \\
\hline $\mathrm{H}$ & 3.82150200 & 1.91219500 & -1.86736500 & $\mathrm{H}$ & 4.44902800 & 2.28748900 & -1.29598500 \\
\hline $\mathrm{H}$ & 4.61187500 & 2.54670900 & -0.42714800 & $\mathrm{H}$ & 4.55048800 & 2.36774800 & 0.44999400 \\
\hline $\mathrm{H}$ & -4.84944900 & 0.15512900 & -0.66403000 & $\mathrm{H}$ & -4.90081300 & 0.03548100 & -0.70393200 \\
\hline $\mathrm{H}$ & -2.48886300 & -3.36542100 & 0.08976000 & $\mathrm{H}$ & -2.49634900 & -3.27880600 & 0.45480200 \\
\hline $\mathrm{H}$ & -2.02845400 & -2.94205400 & -1.55933900 & $\mathrm{H}$ & -2.21042600 & -3.20543700 & -1.28376500 \\
\hline $\mathrm{H}$ & -3.63597200 & -3.65807800 & -1.25021800 & $\mathrm{H}$ & -3.78725000 & -3.77944000 & -0.67387000 \\
\hline C & 1.00870700 & 0.15990600 & 2.18525500 & $\mathrm{H}$ & 5.03403400 & -1.50366100 & -0.39493600 \\
\hline $\mathrm{H}$ & 1.92711700 & -0.30302200 & 2.54873800 & C & 0.932220500 & 0.16724700 & 2.06465000 \\
\hline $\mathrm{H}$ & 1.00145600 & 1.21935000 & 2.44450600 & $\mathrm{H}$ & 1.85601300 & -0.28264100 & 2.43130400 \\
\hline $\mathrm{H}$ & 0.14706100 & -0.32869100 & 2.64278600 & $\mathrm{H}$ & 0.89189100 & 1.21635400 & 2.36128400 \\
\hline $\mathrm{O}$ & -3.28960200 & -0.96490200 & 1.62283400 & $\mathrm{H}$ & 0.07527100 & -0.35796400 & 2.48801400 \\
\hline $\mathrm{H}$ & -2.58641200 & -1.56055900 & 1.92708300 & 0 & -2.78669400 & -1.06002900 & 2.16371200 \\
\hline & & & & $\mathrm{H}$ & -3.21907300 & -0.19249400 & 2.27469300 \\
\hline \multicolumn{8}{|c|}{ Name: UA-C6'-OH-RAF } \\
\hline \multicolumn{8}{|c|}{ Cartesian Coordinates } \\
\hline 0 & -0.61864000 & -1.78337000 & 0.11607500 & 0 & -0.60981600 & -1.74330400 & 0.41490100 \\
\hline $\mathrm{O}$ & 2.08022900 & 1.88214700 & -0.09627800 & $\mathrm{O}$ & 1.96499700 & 1.95896100 & -0.24755600 \\
\hline 0 & -0.21595600 & 2.86815800 & 0.26961300 & 0 & -0.48618100 & 2.92952200 & 0.20258200 \\
\hline 0 & 4.03052700 & -2.44816100 & -0.35558700 & 0 & 4.06933300 & -2.26272300 & 0.07351000 \\
\hline $\mathrm{O}$ & -4.43056500 & 1.05653600 & -0.82099400 & 0 & -4.65483900 & 0.78527000 & -0.14402800 \\
\hline $\mathrm{O}$ & 5.38505400 & -0.21125700 & -1.23098300 & 0 & 5.44273600 & -0.37944400 & -0.44889500 \\
\hline 0 & -4.74879700 & -1.42995900 & -0.91520900 & 0 & -4.83392200 & -1.67164300 & -0.20184400 \\
\hline C & 0.87301100 & -0.02178300 & 0.63424100 & C & 0.77406500 & 0.13016000 & 0.67644100 \\
\hline C & -0.49649200 & 0.49961400 & 0.27401700 & C & -0.58945400 & 0.54397700 & 0.20693500 \\
\hline C & 0.71505000 & -1.44496800 & 0.20814700 & C & 0.69371300 & -1.35020900 & 0.45848700 \\
\hline C & 2.10288800 & 0.62404000 & 0.01940400 & C & 1.99618400 & 0.75603900 & 0.03576100 \\
\hline C & 3.21795900 & -0.20086400 & -0.26434100 & C & 3.15892700 & -0.09323300 & -0.06275900 \\
\hline C & -1.34419600 & -0.60991400 & 0.13858200 & C & -1.34625500 & -0.63403200 & 0.05763400 \\
\hline C & -1.00501000 & 1.78839900 & 0.12121600 & C & -1.21287700 & 1.80459700 & 0.12899400 \\
\hline C & 1.72425000 & -2.24508800 & -0.11752600 & C & 1.74568300 & -2.16147500 & 0.32446700 \\
\hline C & 3.07466100 & -1.65567800 & -0.23265600 & C & 3.04400300 & -1.53245800 & 0.14447600 \\
\hline C & -2.68389000 & -0.50071100 & -0.32185200 & C & -2.75682600 & -0.62591900 & 0.00408800 \\
\hline C & -2.35385400 & 1.96032200 & -0.23277700 & C & -2.59496300 & 1.87534200 & 0.00218300 \\
\hline C & -3.15534000 & 0.82221400 & -0.45600400 & C & -3.34176800 & 0.66550100 & -0.04022500 \\
\hline C & 4.50979600 & 0.40322900 & -0.59982200 & C & 4.42987100 & 0.42193200 & -0.39322500 \\
\hline C & -3.58397800 & -1.63417300 & -0.51828200 & C & -3.59469800 & -1.80291700 & -0.14649600 \\
\hline C & -2.95641000 & 3.32062700 & -0.41054700 & C & -3.32213800 & 3.18389400 & -0.08688300 \\
\hline C & 4.83816900 & 1.80717200 & -0.15138400 & C & 4.72485400 & 1.84921200 & -0.68926400 \\
\hline C & -3.13763600 & -3.03349200 & -0.24273500 & C & -2.99458400 & -3.17016800 & -0.25459200 \\
\hline $\mathrm{H}$ & 1.59833800 & -3.28428000 & -0.39010700 & $\mathrm{H}$ & 1.66681300 & -3.23420100 & 0.21670000 \\
\hline $\mathrm{H}$ & -3.81278100 & 3.44846500 & 0.25558900 & $\mathrm{H}$ & -2.61457100 & 4.00788500 & -0.14146300 \\
\hline
\end{tabular}




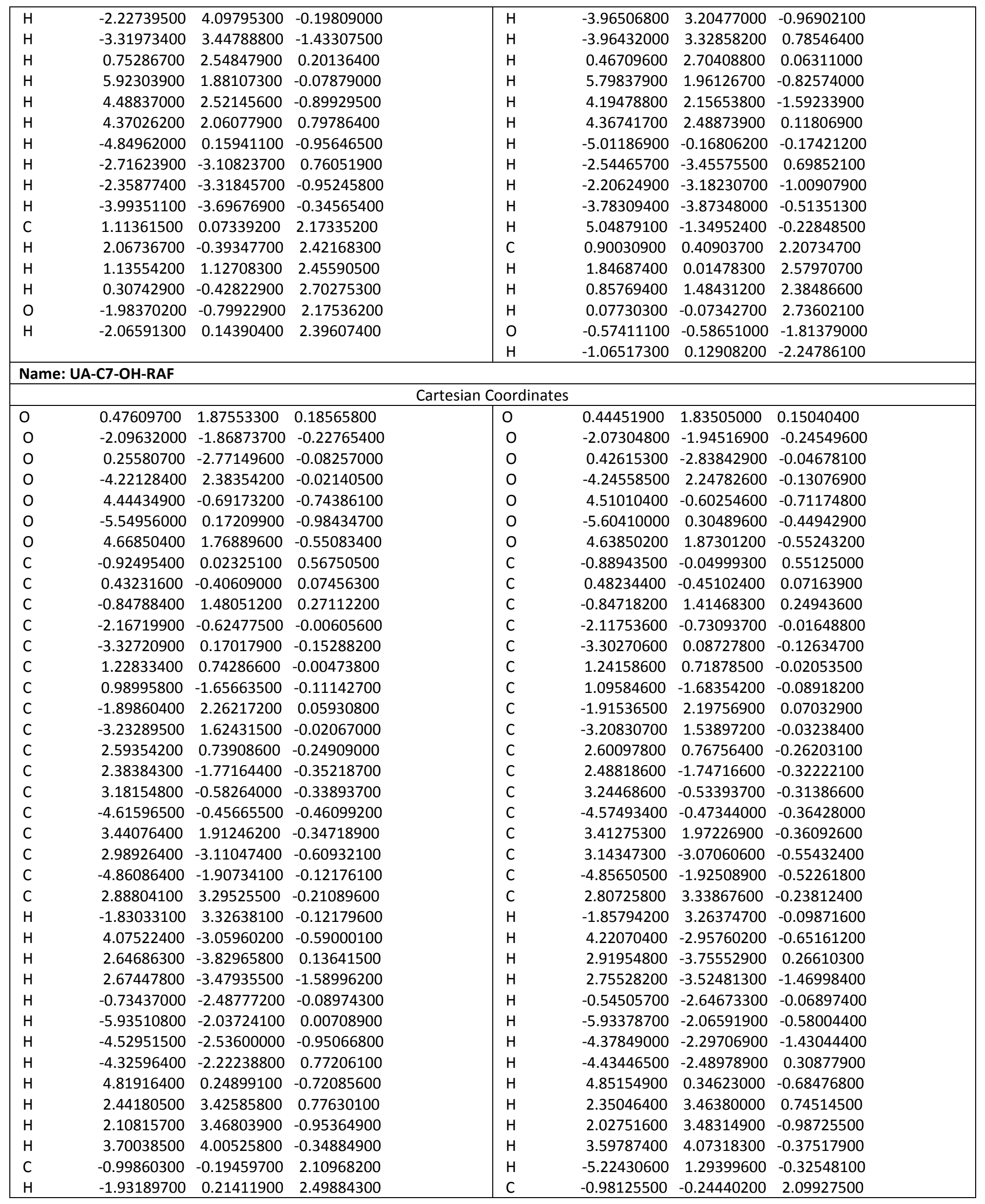




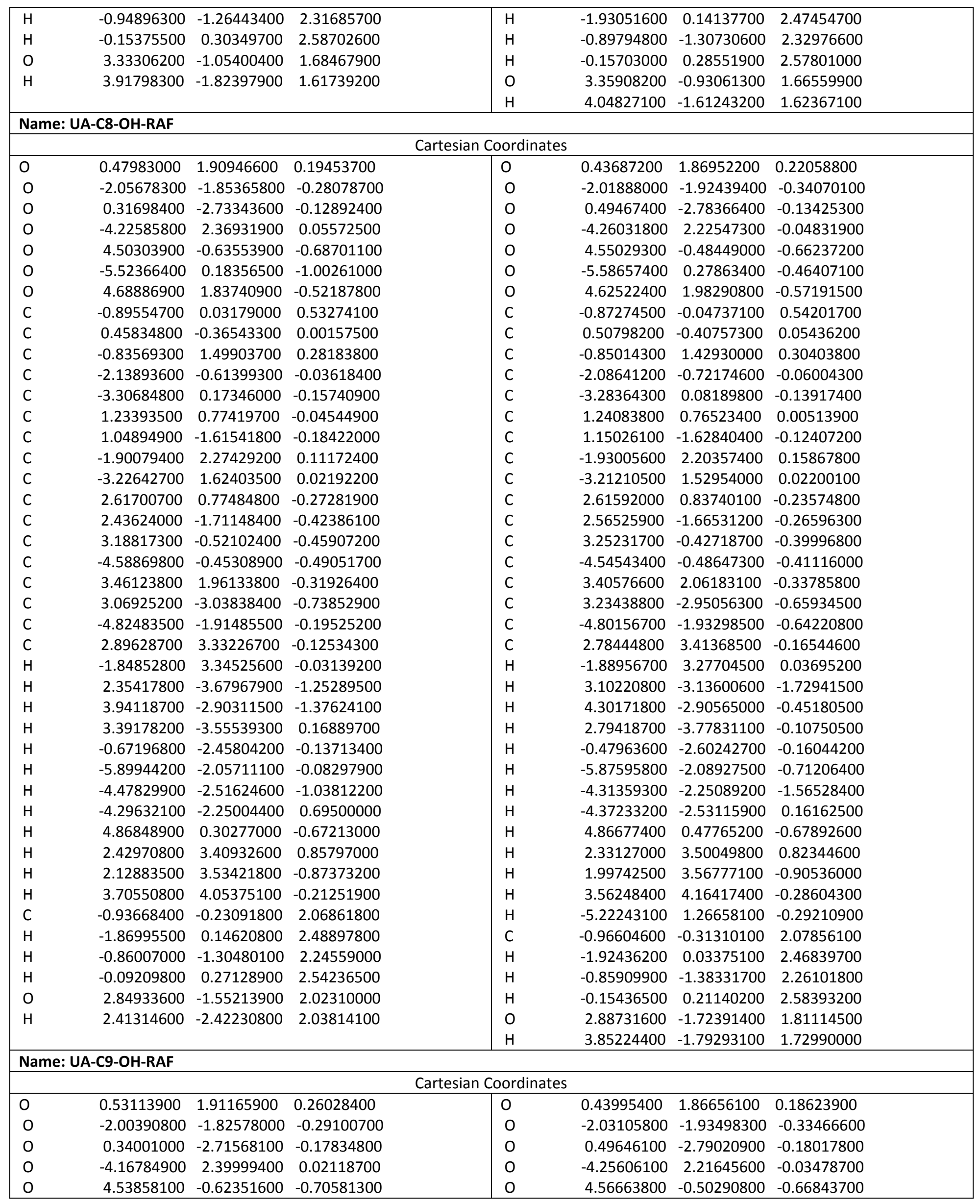




\begin{tabular}{|c|c|c|c|c|c|c|c|}
\hline 0 & -5.43022000 & 0.24693100 & -1.13067300 & 0 & -5.57902100 & 0.31642200 & -0.43108400 \\
\hline 0 & 4.72585700 & 1.83090300 & -0.54679000 & 0 & 4.64426600 & 1.97421000 & -0.52948600 \\
\hline C & -0.87031100 & 0.04907900 & 0.59065600 & C & -0.85973400 & -0.05428400 & 0.50466000 \\
\hline C & 0.50004600 & -0.35879700 & 0.11200800 & C & 0.51208300 & -0.40870900 & -0.01007500 \\
\hline C & -0.78901400 & 1.51482800 & 0.32873700 & C & -0.83953500 & 1.42420200 & 0.28580600 \\
\hline C & -2.09178200 & -0.58683900 & -0.03717700 & C & -2.08906500 & -0.72825500 & -0.06718800 \\
\hline C & -3.25017100 & 0.20481400 & -0.19757000 & C & -3.28124200 & 0.07958900 & -0.12956200 \\
\hline C & 1.27030300 & 0.75961200 & 0.05203100 & C & 1.24451700 & 0.75823500 & -0.04984600 \\
\hline C & 1.10451900 & -1.64395900 & -0.00559800 & C & 1.16116700 & -1.63395300 & -0.19361400 \\
\hline C & -1.84347300 & 2.29710900 & 0.12576300 & C & -1.92808400 & 2.19641900 & 0.17151600 \\
\hline C & -3.16946000 & 1.65379200 & 0.00256500 & C & -3.19361000 & 1.50886600 & 0.03340300 \\
\hline C & 2.67079900 & 0.77250700 & -0.20150700 & C & 2.62167400 & 0.82465400 & -0.27637500 \\
\hline C & 2.49582500 & -1.70756800 & -0.36431200 & C & 2.55186700 & -1.66476400 & -0.40132900 \\
\hline C & 3.24373100 & -0.50062700 & -0.41186300 & C & 3.25319600 & -0.44653800 & -0.44830300 \\
\hline C & -4.51962400 & -0.40755800 & -0.59787100 & C & -4.57315700 & -0.46670900 & -0.39690600 \\
\hline C & 3.50757400 & 1.95918100 & -0.28394100 & C & 3.41779000 & 2.04845100 & -0.33837600 \\
\hline C & 3.15601900 & -3.00555300 & -0.68857200 & C & 3.24918900 & -2.97326400 & -0.64714900 \\
\hline C & -4.77210300 & -1.87550600 & -0.35166400 & C & -4.83209400 & -1.91448200 & -0.63397400 \\
\hline C & 2.95751200 & 3.33118200 & -0.06263300 & C & 2.789890000 & 3.39992300 & -0.17724100 \\
\hline $\mathrm{H}$ & -1.77954700 & 3.36650600 & -0.02422500 & $\mathrm{H}$ & -1.89813900 & 3.27168500 & 0.06544500 \\
\hline $\mathrm{H}$ & 3.44461400 & -3.02438600 & -1.74324700 & $\mathrm{H}$ & 3.15118300 & -3.27534900 & -1.69390100 \\
\hline $\mathrm{H}$ & 4.06506300 & -3.12997000 & -0.09762500 & $\mathrm{H}$ & 4.31129800 & -2.89069200 & -0.42213100 \\
\hline $\mathrm{H}$ & 2.48333600 & -3.83833700 & -0.49666100 & $\mathrm{H}$ & 2.80932800 & -3.75651400 & -0.03073400 \\
\hline $\mathrm{H}$ & -0.65193200 & -2.41213100 & -0.17654500 & $\mathrm{H}$ & -0.47609900 & -2.60656400 & -0.18665900 \\
\hline $\mathrm{H}$ & -5.85119700 & -2.02076700 & -0.30921300 & $\mathrm{H}$ & -5.90549500 & -2.06188000 & -0.73312400 \\
\hline $\mathrm{H}$ & -4.37250900 & -2.45823100 & -1.18394100 & $\mathrm{H}$ & -4.31870400 & -2.23983200 & -1.54019700 \\
\hline $\mathrm{H}$ & -4.29878600 & -2.23029600 & 0.56177400 & $\mathrm{H}$ & -4.43252500 & -2.51275100 & 0.18552400 \\
\hline $\mathrm{H}$ & 4.90301600 & 0.32342800 & -0.71159200 & $\mathrm{H}$ & 4.88838400 & 0.45093500 & -0.65532900 \\
\hline $\mathrm{H}$ & 2.49763400 & 3.40121800 & 0.92392100 & $\mathrm{H}$ & 2.32541200 & 3.48756900 & 0.80641200 \\
\hline $\mathrm{H}$ & 2.18794700 & 3.54961700 & -0.80458500 & $\mathrm{H}$ & 2.01121500 & 3.55237600 & -0.92601200 \\
\hline $\mathrm{H}$ & 3.77184600 & 4.04700700 & -0.14821700 & $\mathrm{H}$ & 3.56835900 & 4.15152500 & -0.28830000 \\
\hline C & -1.00456300 & -0.20109800 & 2.12335300 & $\mathrm{H}$ & -5.10761800 & 1.39880900 & -0.23714900 \\
\hline $\mathrm{H}$ & -1.94119200 & 0.22588000 & 2.48474300 & C & -0.91900000 & -0.32760500 & 2.04352000 \\
\hline $\mathrm{H}$ & -0.99282900 & -1.27469600 & 2.31334700 & $\mathrm{H}$ & -1.87242900 & 0.00668600 & 2.45558400 \\
\hline $\mathrm{H}$ & -0.16574200 & 0.26549500 & 2.64093000 & $\mathrm{H}$ & -0.79802000 & -1.39783200 & 2.21627300 \\
\hline 0 & 1.84055600 & -1.91011400 & 1.84513700 & $\mathrm{H}$ & -0.10264500 & 0.20286600 & 2.53462300 \\
\hline \multirow[t]{2}{*}{$\mathrm{H}$} & 2.13199100 & -2.83311000 & 1.78489400 & 0 & 2.77367500 & -1.60203600 & 2.10775100 \\
\hline & & & & $\mathrm{H}$ & 3.69254900 & -1.85977200 & 1.91304000 \\
\hline
\end{tabular}

